\title{
Design of a robust active fuzzy parallel distributed compensation anti-vibration controller for a hand-glove system
}

\author{
Leila Rajabpour ${ }^{1}$, Hazlina Selamat ${ }^{\text {Corresp., } 1}$, Alireza Barzegar ${ }^{2}$, Mohamad Fadzli Haniff ${ }^{3}$ \\ ${ }^{1}$ School of Electrical Engineering, Universiti Teknologi Malaysia, Johor Bahru, Johor, Malaysia \\ 2 Electrical Engineering, Nanyang Technological University, Singapore, Singapore, Singapore \\ 3 Malaysia-Japan International Institute of Technology, Universiti Teknologi Malaysia, Kuala Lumpur, Federal Territory, Malaysia \\ Corresponding Author: Hazlina Selamat \\ Email address: hazlina@utm.my
}

Undesirable vibrations resulting from the use of vibrating hand-held tools decrease the tool performance and user productivity. In addition, prolonged exposure to the vibration can cause ergonomic injuries known as the hand-arm vibration syndrome (HVAS). Therefore, it is very important to design a vibration suppression mechanism that can isolate or suppress the vibration transmission to the users' hands to protect them from HAVS. While viscoelastic materials in anti-vibration gloves are used as the passive control approach, an active vibration control has shown to be more effective but requires the use of sensors, actuators and controllers. In this paper, the design of a controller for an anti-vibration glove is presented. The aim is to keep the level of vibrations transferred from the tool to the hands within a healthy zone. The paper also describes the formulation of the handglove system's mathematical model and the design of a fuzzy parallel distributed compensation (PDC) controller that can cater for different hand masses. The performances of the proposed controller are evaluated through simulations and the results are benchmarked with two other active vibration control techniques - proportional integral derivative (PID) controller and active force controller (AFC). The simulation results show a superior performance of the proposed controller over the benchmark controllers. The designed PDC controller is able to suppress the vibration transferred to the user's hand 93\% and $85 \%$ better than the PID controller and the AFC, respectively. 


\title{
Design of a robust active Fuzzy parallel distributed compensation anti-vibration controller for a hand-glove system
}

\author{
Leila Rajabpour ${ }^{1}$, Hazlina Selamat ${ }^{1}$, Alireza Barzegar ${ }^{2}$, and Mohamad \\ Fadzli Haniff ${ }^{3}$

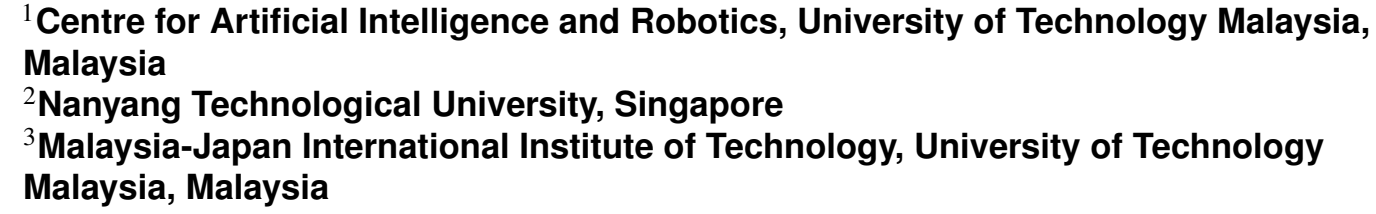

\begin{abstract}
Undesirable vibrations resulting from the use of vibrating hand-held tools decrease the tool performance and user productivity. In addition, prolonged exposure to the vibration can cause ergonomic injuries known as the hand-arm vibration syndrome (HAVS). Therefore, it is very important to design a vibration suppression mechanism that can isolate or suppress the vibration transmission to the users' hands to protect them from HAVS. While viscoelastic materials in anti-vibration gloves are used as the passive control approach, an active vibration control has shown to be more effective but requires the use of sensors, actuators and controllers. In this paper, the design of a controller for an anti-vibration glove is presented. The aim is to keep the level of vibrations transferred from the tool to the hands within a healthy zone. The paper also describes the formulation of the hand-glove system's mathematical model and the design of a fuzzy parallel distributed compensation (PDC) controller that can cater for different hand masses. The performances of the proposed controller are evaluated through simulations and the results are benchmarked with two other active vibration control techniques - proportional integral derivative (PID) controller and active force controller (AFC). The simulation results show a superior performance of the proposed controller over the benchmark controllers. The designed PDC controller is able to suppress the vibration transferred to the user's hand $93 \%$ and $85 \%$ better than the PID controller and the AFC, respectively.
\end{abstract}

\section{INTRODUCTION}

Vibrations of the hand-held tools can cause harmful health conditions among the operators. There have been many investigations on the effects of the long-term vibration exposure on the human body (Shen and House, 2017) and many damages have been reported including HAVS, muscle weakness, white finger, loss of grip strength, sensory nerve damage, and muscle and joint injuries in the hand and arm (Gerhardsson et al., 2020; Vihlborg et al., 2017). The effects of anti-vibration (AV) gloves on reducing the health risks of the vibrating tools have been investigated in many studies, and various types of anti-vibration gloves have been introduced to the market such as gel-filled, air-filled and leather AV gloves (Hamouda et al., 2017).

Various biodynamic models of the hand have been proposed in International Standard ISO 10068:2012 (Dong et al., 2009; Rezali and Griffin, 2018) to investigate the transmissibility of the vibration to the hand. The strategy of building the hand model is based on introducing the simple mechanical damper and spring elements, which are arranged in series or parallel depending on the approach used such as Maxwell or Kelvin models of muscles (Jones, 2001), to represent the viscoelastic characteristics of the hand soft tissues. The factors such as types of tools used, the points at which vibrations are transmitted (e.g. the 
hand or the arm) and the required levels of accuracy determine the modeling approach to be used. For example, in (Mazlan and Ripin, 2015) a two degree of freedom (DOF) model was adopted while some higher DOF models can be found in (Kamalakar and Mitra, 2018). A precise representation of different impulsive tools and their working postures and the directions of the applied hand force is illustrated in (Dong et al., 2015).

Incorporating a glove into the hand system requires additional mechanical damper and spring elements in the equivalent mechanical system. For example, the model proposed by Dong (Dong et al., 2009) is a 7-DOF model that represents the viscous, elastic and inertial properties of the glove by the equivalent springs and dampers.

Adding an active element to the passive anti-vibration glove can improve the efficacy of the system. In active vibration control (AVC), an actuator is utilized to apply an external force or displacement based on the measurement of the system response through feedback control (Preumont, 2018). The acceleration, displacement and velocity measurements from the sensors are used by the control system to provide control signals for actuators based on the chosen control strategy.

The design of the control scheme could be quite challenging in this area since many parameters are affecting the performance of the controller such as sensor and actuator's fault (Gao and Liu, 2020; Tahoun, 2020) and uncertainties in the system parameters (Chen et al., 2019; Li et al., 2017; Tahoun, 2017). Besides, the vibration of different body parts occurs at different frequencies and therefore would add complexity to the control design process (Hassan et al., 2010).

Various types of control schemes have been developed and employed in AVC structures. Each new control method has been designed for a specific system with a specific dynamics, such as integral-based controllers in (Zuo and Wong, 2016), sliding mode controls (Hamzah et al., 2012; Lin et al., 2019), PID control (Rani et al., 2011), AFC (Theik and Mazlan, 2020) and artificial intelligent-based control strategies including fuzzy and neural networks (Kalaivani et al., 2016). In (Lekshmi and Ramachandran, 2019) a Genetic Algorithm-optimized PID controller is proposed to suppress Parkinson's Tremor. In (Liu et al., 2018), an adaptive neural network controller is proposed to control the suspension systems, and some fuzzy active suspension system controllers are also proposed in (Sun et al., 2018; Tabatabaei et al., 2010).

The AFC proposed in (Zain et al., 2008) is an effective method designed to carry out the robust control of dynamic systems in the presence of disturbance and uncertainties. It is proved that in AFC, the stability and robustness of the system remain unchanged by compensation action of the control strategy when a number of disturbances are applied to the system (Mailah et al., 2009).

The research on fuzzy systems and control has resulted in the development of many controller designs especially by using the Takagi-Sugeno (T-S) fuzzy model as an effective and simple tool in modeling and control of nonlinear systems or systems with variable parameters (Rajabpour et al., 2019; Sadeghi et al., 2016). Additionally, The T-S model can generate an exact representation of the nonlinear system by a set of linear subsystems. Since the hand mass is variable for different users in the hand-glove system, we propose to use the fuzzy T-S model for the standard range of various hand masses and employing the idea of fuzzy parallel distributed compensation (PDC) technique, proposed by Wang et al. (Wang et al., 1995), to control the fuzzy T-S modeled systems by designing a linear feedback controller for each linear subsystem.

Originally, the idea of PDC has been applied to control nonlinear systems by linearizing them around different operation points. Here, we used this idea to control the linear hand-glove system but we used the ability of PDC control to make the model and controller more flexible for different users with different hand masses which has not been considered in the previous controller designs. Building on the anti-vibration gloves literature, we propose the design of an active anti-vibration controller for the hand-glove system to suppress the transmitted vibration to the hand while using hand-held tools. To our knowledge, it is the first anti-vibration glove with an active controller. In this paper, the mathematical model of the hand-glove system is formulated and three different controllers- a PID controller, an AFC and a fuzzy PDC controller- are designed and applied to the hand-glove system. The performances of the three controllers are then compared.

To summarize, the key contributions of this paper are twofold: the T-S fuzzy modeling of the handglove system for variable hand mass parameter and the design of an active anti-vibration glove based on the fuzzy PDC controller, which is robust to variation in hand masses, making the active anti-vibration glove suitable for different users.

This paper is organized as follows: Section 1 presents the mathematical formulation of the hand-glove 
model. Section 2 describes the development of the fuzzy PDC active anti-vibration controller for the hand-glove system. Section 3 demonstrates the simulation results and analysis and the Section 4 concludes the paper.

\section{MODELLING OF ACTIVE HAND-GLOVE SYSTEM}

To represent the dynamic behavior of the hand-glove system under the influence of vibration, the system is represented by a three-degree of freedom model as represented in Figure 1. All the masses are assumed to be rigid and the mass of each segment is considered to be a percentage of the total body mass (Rezali and Griffin, 2018). Since our main focus is on the transmitted vibration to the hand and to reduce the complexity of the model, the palm and fingers are considered to be one body segment and the palm is assumed to be lying on the vibration surface. Table 1 lists the symbols, units and descriptions of the parameters of the hand-glove system shown in Figure 1.

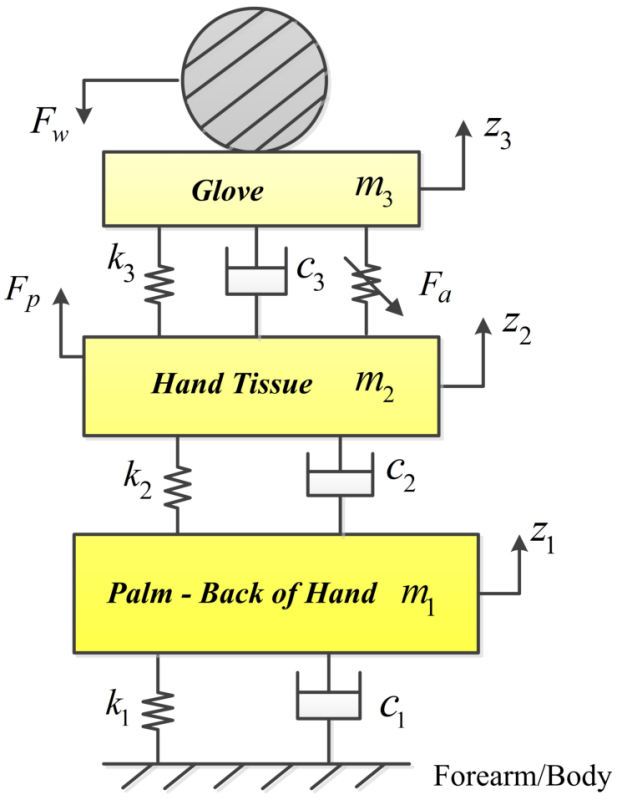

Figure 1. The three DOF hand-glove model

\begin{tabular}{c|l|c} 
Symbol & Description & Unit \\
\hline$m_{1}$ & Mass of the palm and back of hand & $\mathrm{kg}$ \\
$m_{2}$ & Mass of the hand tissue & $\mathrm{kg}$ \\
$m_{3}$ & Mass of the glove & $\mathrm{kg}$ \\
$c_{1}$ & Stiffness coefficient of the hand & $\mathrm{N} / \mathrm{m}$ \\
$c_{2}$ & Stiffness coefficient properties of the soft tissue of the hand & $\mathrm{N} / \mathrm{m}$ \\
$c_{3}$ & Stiffness coefficient properties of the glove material & $\mathrm{N} / \mathrm{m}$ \\
$k_{1}$ & Damping coefficient of the hand & $\mathrm{N.s} / \mathrm{m}$ \\
$k_{2}$ & Damping coefficient of the soft tissue of the hand & $\mathrm{N.s} / \mathrm{m}$ \\
$k_{3}$ & Damping coefficient of the glove material & $\mathrm{N.s} / \mathrm{m}$ \\
$z_{i}$ & Displacement of mass $m_{i}$ & $\mathrm{~m}$ \\
$F_{a}$ & Control Input (Actuatorforce) & $\mathrm{N}$ \\
$F_{w}$ & Vibration Input (Disturbance) & $\mathrm{m} / \mathrm{a}^{2}$ \\
$F_{p}$ & Push force & $\mathrm{N}$
\end{tabular}

Table 1. Gloved-hand model parameters. 


\subsection{The Equations of Motion of the Active Hand-Glove Model}

In Figure 1, since the actuator is assumed to be placed on the palm under the glove, the actuator force $F_{a}$ is applied between the soft tissue of the hand, mass $m_{2}$ and glove material with mass $m_{3}$. The hand pushing the tool handle is represented by $F_{p}$.

The dynamic equations of motion of the system can be written as follows:

$$
\begin{aligned}
& m_{1}(t) \ddot{z}_{1}+\left(c_{1}+c_{2}\right) \dot{z}_{1}-c_{2} \dot{z}_{2}+\left(k_{1}+k_{2}\right) z_{1}-k_{2} z_{2}=0 \\
& m_{2} \ddot{z}_{2}-c_{2} \dot{z}_{1}+\left(c_{2}+c_{3}\right) \dot{z}_{2}-c_{3} \dot{z}_{3}-k_{2} z_{1}+\left(k_{2}+k 3\right) z_{2} \\
& -k_{3} z_{3}=F_{a}-F_{p} \\
& m_{3} \ddot{z}_{3}-c_{3} \dot{z}_{2}+c_{3} \dot{z}_{3}-k_{3} z_{2}+k_{3} z_{3}=-F_{a}+F_{w}
\end{aligned}
$$

where $z_{i}, \dot{z}_{i}$ and $\ddot{z}_{i}$ represent the displacement, velocity and acceleration of mass $m_{i} ; F_{a}$ is the actuator force, $F_{p}$ is the push force and $F_{w}$ is the input vibration or disturbance to the system that needs to be controlled.

In this model, the mass of the hand is assumed to be non-constant, $m_{1}(t)$, i.e. the model can be used to represent the hand system for different users. The equations (1) can be arranged in the following form:

$$
\begin{aligned}
& \ddot{z}_{1}=-\frac{c_{1}+c_{2}}{m_{1}(t)} \dot{z}_{1}+\frac{c_{2}}{m_{1}(t)} \dot{z}_{2}-\frac{k_{1}+k_{2}}{m_{1}(t)} z_{1}+\frac{k_{2}}{m_{1}(t)} z_{2} \\
& \ddot{z}_{2}=\frac{c_{2}}{m_{2}} \dot{z}_{1}-\frac{c_{2}+c_{3}}{m_{2}} \dot{z}_{2}+\frac{c_{3}}{m_{2}} \dot{z}_{3}+\frac{k_{2}}{m_{2}} z_{1}-\frac{k_{2}+k_{3}}{m_{2}} z_{2}+\frac{k_{3}}{m_{2}} z_{3}+\frac{F_{a}}{m_{2}}-\frac{F_{p}}{m_{2}} \\
& \ddot{z}_{3}=\frac{c_{3}}{m_{3}} \dot{z}_{2}-\frac{c_{3}}{m_{3}} \dot{z}_{3}+\frac{k_{3}}{m_{3}} z_{2}-\frac{k_{3}}{m_{3}} z_{3}-\frac{F_{a}}{m_{3}}+\frac{F_{w}}{m_{3}}
\end{aligned}
$$

Considering $x_{1}=z_{1}, x_{2}=z_{2}, x_{3}=z_{3}, x_{4}=\dot{z}_{1}, x_{5}=\dot{z}_{2}$ and $x_{6}=\dot{z}_{3}$ for each of the state variables in equation (2), it can be rewritten in the state-space equation given by Equation (3).

$$
\begin{aligned}
& \dot{\mathbf{x}}(t)=\mathbf{A}(t) \mathbf{x}(t)+\mathbf{B}(t) \mathbf{u}(t)+\mathbf{E}(t) \mathbf{w}(t) \\
& \mathbf{y}(t)=\mathbf{C} \mathbf{x}(t)
\end{aligned}
$$

where matrices $\mathbf{A}, \mathbf{B}, \mathbf{C}$ and $\mathbf{E}$ are the system matrix, the input matrix, output matrix and the disturbance matrix, respectively. The hand vibration, $\dot{x}_{4}$ or $\ddot{z}_{1}$ is considered as the performance output reference. Equation (3) can be further written in the following form:

$$
\begin{aligned}
& {\left[\begin{array}{c}
\dot{x}_{1} \\
\dot{x}_{2} \\
\dot{x}_{3} \\
\dot{x}_{4} \\
\dot{x}_{5} \\
\dot{x}_{6}
\end{array}\right]=\left[\begin{array}{cccccc}
0 & 0 & 0 & 1 & 0 & 0 \\
0 & 0 & 0 & 0 & 1 & 0 \\
0 & 0 & 0 & 0 & 0 & 1 \\
-\frac{k_{1}+k_{2}}{m_{1}(t)} & \frac{k_{2}}{m_{1}(t)} & 0 & -\frac{c_{1}+c_{2}}{m_{1}(t)} & \frac{c_{2}}{m_{1}(t)} & 0 \\
\frac{k_{2}}{m_{2}} & -\frac{k_{2}+k_{3}}{m_{2}} & \frac{k_{3}}{m_{2}} & \frac{c_{2}}{m_{2}} & -\frac{c_{2}+c_{3}}{m_{2}} & \frac{c_{3}}{m_{2}} \\
0 & \frac{k_{3}}{m_{3}} & -\frac{k_{3}}{m_{3}} & 0 & \frac{c_{3}}{m_{3}} & -\frac{c_{3}}{m_{3}}
\end{array}\right]\left[\begin{array}{l}
x_{1} \\
x_{2} \\
x_{3} \\
x_{4} \\
x_{5} \\
x_{6}
\end{array}\right]} \\
& +\left[\begin{array}{cc}
0 & 0 \\
0 & 0 \\
0 & 0 \\
0 & 0 \\
\frac{1}{m_{2}} & -\frac{1}{m_{2}} \\
-\frac{1}{m_{3}} & 0
\end{array}\right]\left[\begin{array}{c}
F_{a} \\
F_{p}
\end{array}\right]+\left[\begin{array}{c}
0 \\
0 \\
0 \\
0 \\
0 \\
\frac{1}{m_{3}}
\end{array}\right] F_{w} \\
& y(t)=\left[\begin{array}{llllll}
0 & 0 & 0 & 1 & 0 & 0
\end{array}\right]\left[\begin{array}{llllll}
x_{1} & x_{2} & x_{3} & x_{4} & x_{5} & x_{6}
\end{array}\right]^{T}
\end{aligned}
$$

Another approach that can be used to model the active hand-glove system is the fuzzy T-S model and is described next. 


\subsection{Fuzzy Takagi-Sugeno Model}

Fuzzy logic has the ability to deal with nonlinearities and uncertainties in the system where standard analytical models are usually ineffective. It can be used for both modeling and design of the controller. Most nonlinear systems can be approximated by a T-S fuzzy model. The T-S fuzzy model of a dynamic system with control input and disturbance input can be represented by the following rules:

Model Rule i:

IF $s_{1}(t)$ is $M_{i 1}$ and $\cdots$ and $s_{p}(t)$ is $M_{i p}$,

$$
\text { THEN }\left\{\begin{aligned}
\dot{\mathbf{x}}(t) & =\mathbf{A}_{i} \mathbf{x}(t)+\mathbf{B}_{i} \mathbf{u}(t)+\mathbf{E}_{i} \mathbf{w}(t), \quad i=1,2, \ldots, r, \\
\mathbf{y}(t) & =\mathbf{C}_{i} \mathbf{x}(t),
\end{aligned}\right.
$$

where $r$ shows the number of rules, $M_{i j}$ denotes the fuzzy set, and $\mathbf{x}(t) \in \mathbf{R}^{n \times n}, \mathbf{u}(t) \in \mathbf{R}^{n \times 1}$ and $\mathbf{w}(t) \in \mathbf{R}^{n \times 1}$ are state vectors, controlled input vector and disturbance input vector, respectively. Note that $s_{i}(t)$ is a hypothetical variable which can be a function of state variables, disturbance inputs, system parameters and/or time.

By considering each set of $\left(\mathbf{A}_{i}, \mathbf{B}_{i}, \mathbf{E}_{i}, \mathbf{C}_{i}\right)$ as a subsystem, the overall output of the fuzzy system can be represented as

$$
\begin{aligned}
\dot{\mathbf{x}}(t) & =\frac{\sum_{i=1}^{r} \alpha_{i}(\mathbf{s}(t))\left(\mathbf{A}_{i} \mathbf{x}(t)+\mathbf{B}_{i} \mathbf{u}(t)+\mathbf{E}_{i} \mathbf{w}(t)\right)}{\sum_{i=1}^{r} \alpha_{i}(\mathbf{s}(t))} \\
& =\sum_{i=1}^{r} h_{i}(\mathbf{s}(t))\left(\mathbf{A}_{i} \mathbf{x}(t)+\mathbf{B}_{i} \mathbf{u}(t)+\mathbf{E}_{i} \mathbf{w}(t)\right), \\
\mathbf{y}(t) & =\frac{\sum_{i=1}^{r} \alpha_{i}(\mathbf{s}(t))\left(\mathbf{C}_{i} \mathbf{x}(t)\right)}{\sum_{i=1}^{r} \alpha_{i}(\mathbf{s}(t))} \\
& =\sum_{i=1}^{r} h_{i}(\mathbf{s}(t))\left(\mathbf{C}_{i} \mathbf{x}(t)\right),
\end{aligned}
$$

in which for all $t$

$$
\begin{aligned}
& \mathbf{s}(t)=\left[\begin{array}{llll}
s_{1}(t) & s_{2}(t) \ldots s_{p}(t)
\end{array}\right] \\
& \alpha_{i}(\mathbf{s}(t))=\prod_{j=1}^{v} M_{i j}\left(s_{j}(t)\right)
\end{aligned}
$$

and

$$
h_{i}(\mathbf{s}(t))=\frac{\alpha_{i}(\mathbf{s}(t))}{\sum_{i=1}^{r} \alpha_{i}(\mathbf{s}(t))} .
$$

Note that the term $M_{i j}\left(s_{j}(t)\right)$ shows the membership grade of $s_{j}(t)$ in $M_{i}$. Also, since $\alpha_{i}(\mathbf{s}(t)) \geq 0$ and $\sum_{i=1}^{r} \alpha_{i}(s(t)) \geq 0$ for $i=1,2, \ldots, r$, then we have $h_{i}(s(t)) \geq 0$ and $\sum_{i=1}^{r} h_{i}(s(t))=1$ for all $t$.

Next, different types of active vibration control techniques applied to the system to suppress the vibration to the desired level are described.

\section{ACTIVE-VIBRATION CONTROLLER (AVC) DESIGN}

In AVC, the control objective is to attenuate the undesired vibration to the desired level by use of an actuator, which could be a piezoelectric device or an electric motor. The incoming vibration is sensed by using a sensing mechanism and the actuator reacts to these vibrations by producing a cancelation signal. The sensors used in AVC are mainly of piezoelectric type (Miljković, 2009). Figure 2 shows 


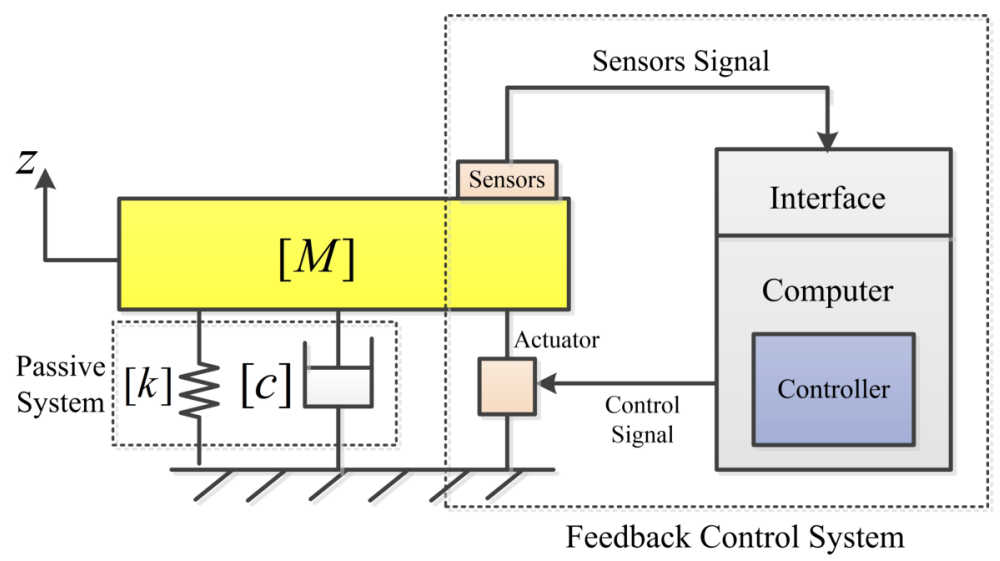

Figure 2. Active vibration control diagram

the arrangement of the AVC mechanism. The vibration cancelation process can be achieved by using different feedforward and feedback controllers. In this figure, $[M]$ is the mass matrix, matrix $[c]$ contains the system damping coefficients and $[k]$ contains the stiffness coefficients of the system.

The working principle of an AVC system is based on collecting data from sensing devices and then generating a control signal for the actuator. The control signal is generated from the measurements of the displacement, velocity or acceleration of the mass $M$ that is fed back. Based on these measurements, the control signal produced depends on the chosen control strategy or algorithm. The followings explain three different control algorithms applied to the hand-glove system to suppress vibrations transmitted from the tools used to the workers' hands.

\subsection{Proportional-Integral-Derivative (PID) Controller}

The PID controller to be used in the AVC system is is a standard PID control algorithm given by Equation (11):

$$
u(t)=K_{p} e(t)+K_{i} \int e(t) d t+K_{d} \dot{e}(t)
$$

where $u(t)$ is the control signal, $e(t)$ is the error signal and $K_{p}, K_{i}$ and $K_{d}$ are the proportional, integral and derivative gains, respectively. Figure 3 shows the feedback control system employing the PID controller. Equation (11) can be written in the following transfer function form:

$$
U(s)=K_{p}+K_{i} / s+K_{d} s
$$

The PID gains $K_{p}, K_{i}$ and $K_{d}$ need to be tuned, by trial and error tuning method, since we get the desired response.

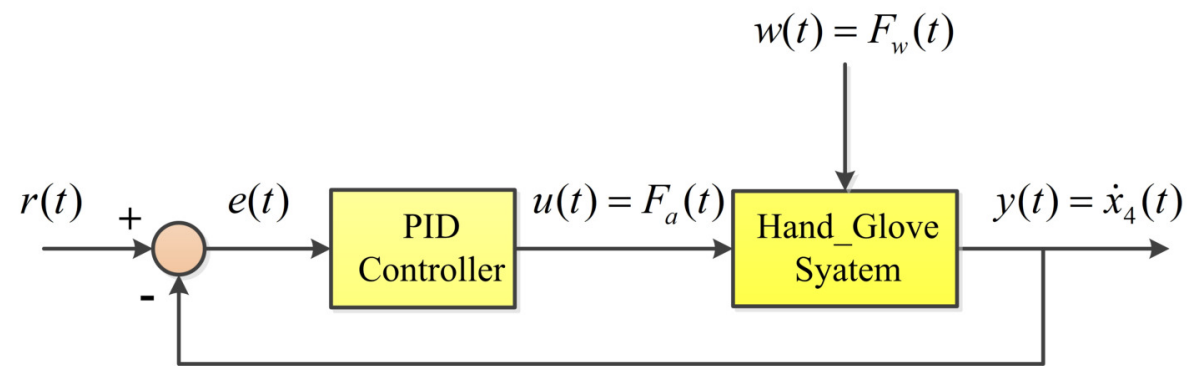

Figure 3. PID control schematic 


\subsection{Active Force Controller}

Active force control (AFC) proposed in (Zain et al., 2008) is an effective method designed to carry out the robust control of dynamic systems in the presence of disturbance and uncertainties. It is proved that in $\mathrm{AFC}$, the stability and robustness of the system remain unchanged by compensation action of the control strategy when a number of disturbances are applied to the system (Abdelmaksoud et al., 2020; Gohari and Tahmasebi, 2017).

The schematic of the AFC controller for a suspension system, which has a similar representation of the hand-glove system, is shown in (Mailah et al., 2009).

One practical example of applying AFC to a suspension system to control the unwanted vibration is given in (Mohamad et al., 2006) and it showed the effectiveness of the applied AFC to cancel the vibration with different disturbances levels.

\subsection{Fuzzy Parallel Distributed Compensation (PDC) Controller}

Fuzzy logic is an effective way to decompose a nonlinear system control into a group of local linear controls based on a set of design-specific model rules. PDC is one of the many varieties of fuzzy logic that can be implemented in control systems. For a given fuzzy T-S framework, the state feedback based on PDC is usually applied. The key idea of the PDC technique is to divide the nonlinear system to some linear subsystems and then design some controllers for each of the linear subsystems and subsequently obtain the overall controller by the fuzzy blending of the local controllers (Sadeghi et al., 2014, 2015; Nguyen et al., 2016) i.e. designing a compensator for each rule of the fuzzy model.

The block diagram of the system with fuzzy PDC controller is illustrated in Figure 4. As can be seen, the overall PDC controller is applied to the original nonlinear system.

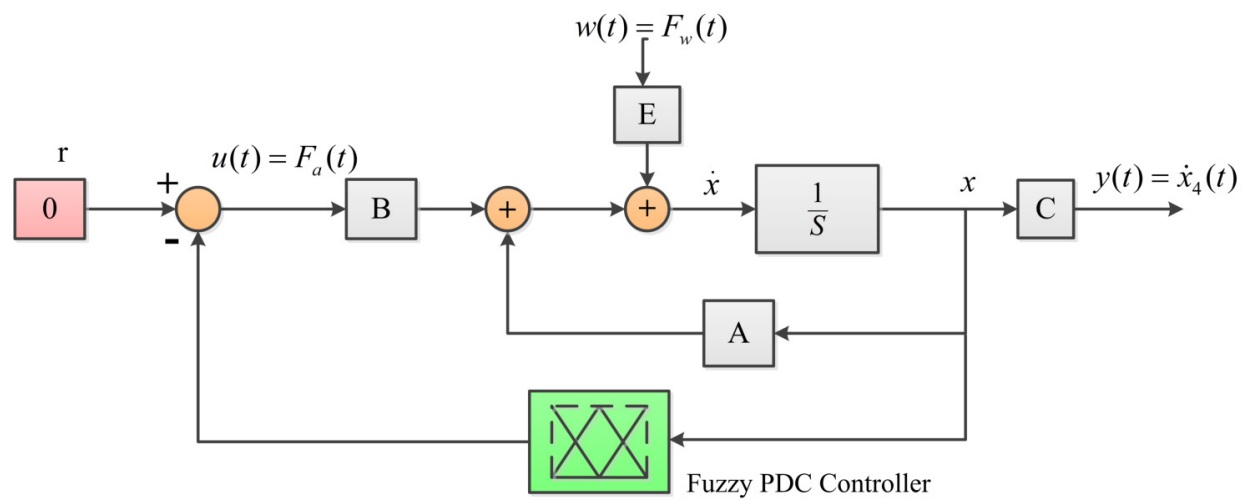

Figure 4. The closed-loop system with fuzzy PDC controller

The PDC technique proposes a method to design a fuzzy controller associated with the fuzzy T-S model Equation (7). In PDC, $r$ controllers, usually state feedback, associated with each rule $i$ are designed as

Control Rule i:

$$
\text { IF } s_{1}(t) \text { is } M_{i 1} \text { and } \cdots \text { and } s_{p}(t) \text { is } M_{i p} \text {, }
$$$$
\text { THEN } u(t)=-F_{i} x(t), \quad i=1,2, \ldots, r .
$$

The overall control output of the fuzzy PDC controller is then obtained as

$$
\begin{aligned}
u(t) & =-\frac{\sum_{i=1}^{r} \alpha_{i}(s(t))\left(F_{i} x(t)\right)}{\sum_{i=1}^{r} \alpha_{i}(s(t))} \\
& =-\sum_{i=1}^{r} h_{i}(s(t))\left(F_{i} x(t)\right),
\end{aligned}
$$


Note that although in the PDC approach the controllers are local (Equation(14)), the overall controller scheme should be designed globally to ensure global stability and suitable control performance. By substituting Equation (14) in (7) the closed loop system can be expressed as

$$
\dot{x}(t)=\sum_{i=1}^{r} \sum_{j=1}^{r} h_{i}(s(t)) h_{j}(s(t))\left(\left(A_{i}-B_{i} F_{j}\right) x(t)+E_{i} w(t)\right) .
$$

Now, by considering a positive definite matrix $P$ and the quadratic Lyapunov function $V(x)=$ $x^{T}(t) P x(t)$, the closed-loop system (15) is asymptotically stable if the following condition is satisfied (Tanaka and Wang, 2004)

$$
\left\{A_{i}-B_{i} F_{j}\right\}^{T}\left\{A_{i}-B_{i} F_{j}\right\}-P<0 \quad \forall i, j=1,2, \ldots, r,
$$

for $h_{i}(s(t)) h_{j}(s(t)) \neq 0, \forall t$.

To design a PDC controller for the hand-glove system, Equation (4)-(5), it is required to obtain the T-S fuzzy model of the dynamic system (4). Since our goal is to design an active controller that guarantees suitable performance for different users, i.e. different hand masses $m_{1}$, it is supposed that $m_{1} \in\left[m_{1}^{\min }, m_{1}^{\max }\right]$. By defining the premise variable $\psi_{h}(t)$ as

$$
\psi_{h}(t):=\frac{1}{m_{1}(t)}
$$

and using the definition in Equation (17) and the possible range of $m_{1}(t)$, the minimum and maximum range of $\psi_{h}(t)$ can be obtained as

$$
\begin{aligned}
& \psi_{h}^{\min }=\min \left\{\frac{1}{m_{1}(t)}\right\}=\frac{1}{m_{1}^{\max }}, \\
& \psi_{h}^{\max }=\max \left\{\frac{1}{m_{1}(t)}\right\}=\frac{1}{m_{1}^{\min }} .
\end{aligned}
$$

Then $\psi_{h}(t)$ can be written in terms of $\psi_{h}^{\min }$ and $\psi_{h}^{\max }$ as follows:

$$
\psi_{h}(t)=M_{1}\left(\psi_{h}(t)\right) \frac{1}{m_{1}^{\min }}+M_{2}\left(\psi_{h}(t)\right) \frac{1}{m_{1}^{\max }}
$$

where

$$
M_{1}\left(\psi_{h}(t)\right)+M_{2}\left(\psi_{h}(t)\right)=1 .
$$

By using Equations (19) and (20), the membership functions can be obtained as follows:

$$
\begin{gathered}
M_{1}\left(\psi_{h}(t)\right)=\frac{\psi_{h}(t)-\frac{1}{m_{1}^{\max }}}{\frac{1}{m_{1}^{\min }}-\frac{1}{m_{1}^{\max }}}, \\
M_{2}\left(\psi_{h}(t)\right)=\frac{\frac{1}{m_{1}^{\min }}-\psi_{h}(t)}{\frac{1}{m_{1}^{\min }}-\frac{1}{m_{1}^{\max }}}
\end{gathered}
$$

which are demonstrated in Figure 5. Note that the membership functions $M_{1}$ and $M_{2}$ are called "Small" and "Large", respectively.

Then, the hand-glove system model Equation (4) can be represented as the following fuzzy T-S model for variable hand masses: 


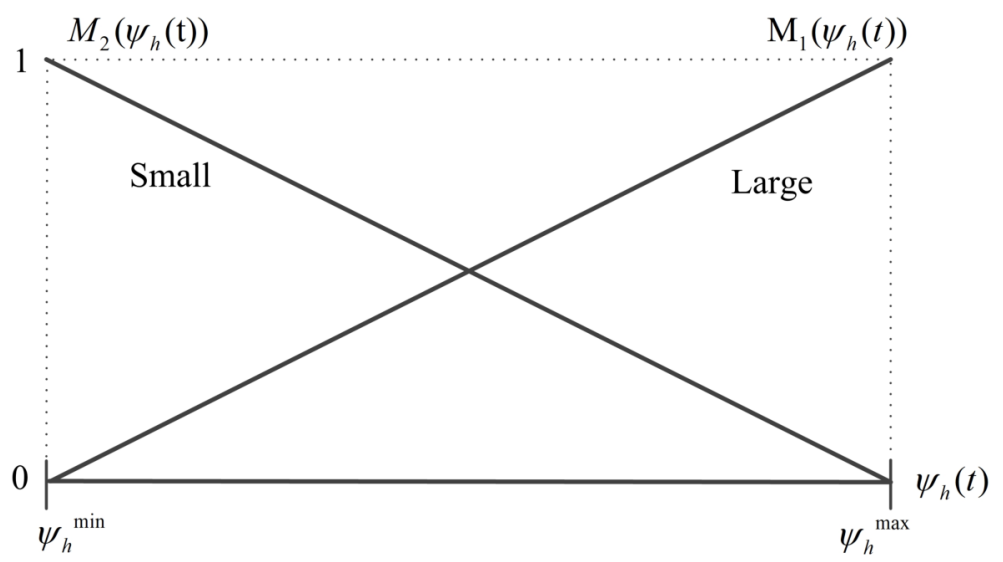

Figure 5. Membership Functions $M_{1}\left(\psi_{h}(t)\right)$ and $M_{2}\left(\psi_{h}(t)\right)$.

215

216

217

218

219

Model Rule 2:

IF $\psi_{h}(t)$ is "Large",

THEN $\left\{\begin{array}{l}\dot{x}(t)=A_{2} x(t)+B_{2} u(t)+E_{2} w(t) \\ y(t)=C_{2} x(t)\end{array}\right.$

where the fuzzy system's submatrices associated with (22a) and (22b) are obtained as

$$
\begin{aligned}
& A_{1}=\left[\begin{array}{cccccc}
0 & 0 & 0 & 1 & 0 & 0 \\
0 & 0 & 0 & 0 & 1 & 0 \\
0 & 0 & 0 & 0 & 0 & 1 \\
-\frac{k_{1}+k_{2}}{m_{1}^{\min }} & \frac{k_{2}}{m_{1}^{\min }} & 0 & -\frac{c_{1}+c_{2}}{m_{1}^{\min }} & \frac{c_{2}}{m_{1}^{\min }} & 0 \\
\frac{k_{2}}{m_{2}} & -\frac{k_{2}+k_{3}}{m_{2}} & \frac{k_{3}}{m_{2}} & \frac{c_{2}}{m_{2}} & -\frac{c_{2}+c_{3}}{m_{2}} & \frac{c_{3}}{m_{2}} \\
0 & \frac{k_{3}}{m_{3}} & -\frac{k_{3}}{m_{3}} & 0 & \frac{c_{3}}{m_{3}} & -\frac{c_{3}}{m_{3}}
\end{array}\right] \\
& A_{2}=\left[\begin{array}{cccccc}
0 & 0 & 0 & 1 & 0 & 0 \\
0 & 0 & 0 & 0 & 1 & 0 \\
0 & 0 & 0 & 0 & 0 & 1 \\
-\frac{k_{1}+k_{2}}{m_{1}^{\max }} & \frac{k_{2}}{m_{1}^{\max }} & 0 & -\frac{c_{1}+c_{2}}{m_{1}^{\max }} & \frac{c_{2}}{m_{1}^{\max }} & 0 \\
\frac{k_{2}}{m_{2}} & -\frac{k_{2}+k_{3}}{m_{2}} & \frac{k_{3}}{m_{2}} & \frac{c_{2}}{m_{2}} & -\frac{c_{2}+c_{3}}{m_{2}} & \frac{c_{3}}{m_{2}} \\
0 & \frac{k_{3}}{m_{3}} & -\frac{k_{3}}{m_{3}} & 0 & \frac{c_{3}}{m_{3}} & -\frac{c_{3}}{m_{3}}
\end{array}\right] \\
& B_{1}=B_{2}=\left[\begin{array}{llllll}
0 & 0 & 0 & 0 & \frac{1}{m_{2}} & -\frac{1}{m_{3}} \\
0 & 0 & 0 & 0 & -\frac{1}{m_{2}} & 0
\end{array}\right]^{T}, \\
& E_{1}=E_{2}=\left[\begin{array}{llllll}
0 & 0 & 0 & 0 & 0 & \frac{1}{m_{3}}
\end{array}\right]^{T},
\end{aligned}
$$




$$
C_{1}=C_{2}=\left[\begin{array}{llllll}
0 & 0 & 0 & 1 & 0 & 0
\end{array}\right]
$$

The designed fuzzy controller shares the same input variables and fuzzy sets with the T-S fuzzy model in the IF parts. So, for the fuzzy models given in Equation (22), the following controller rules are designed via PDC:

Control Rule 1:

IF $\psi_{h}(t)$ is "Small",

THEN $u(t)=-F_{1} x(t)$

Control Rule 2:

IF $\psi_{h}(t)$ is "Large",

THEN $u(t)=-F_{2} x(t)$

where $F_{1}$ and $F_{2}$ are the feedback gains.

Now, by considering that the disturbance input, i.e. vibration from the hand-held tool, has a limited energy it can be assumed that $w \in L_{2}[0, \infty)$ and $\|w\|_{2}^{2} \leq w_{\max }$. Then, the following control performances are aimed to be obtained:

1. The closed-loop system remains stable.

2. The transmitted vibration to the hand will be reduced significantly, i.e. the effect of disturbance input in the system output will be minimized or mathematically

$$
\|y\|_{2}^{2}<\gamma\|w\|_{2}^{2}
$$

for all $w \neq 0$ where $\gamma$ is a predefined scalar.

3. The designed control input is enforced to a limited value, i.e.

$$
\|u\|_{2} \leq u^{\max } .
$$

4. The designed controller performs well for different users/hand masses.

Theorem: The feedback gains, $F_{i}$, of the state feedback controller (24) that stabilize the fuzzy T-S system (22) while minimizing the $\gamma$ in (25) and satisfying the control effort constraint (26) can be obtained by solving the following optimization linear matrix inequality (LMI)-based problem as follows:

$$
\begin{aligned}
& \min _{\Gamma, M_{1}, \ldots, M_{r}} \gamma^{2} \\
& \text { subject to }
\end{aligned}
$$

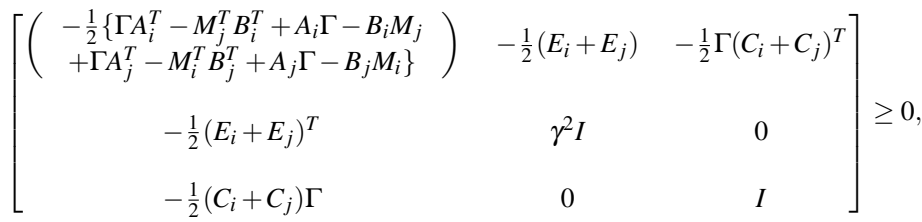

$$
\begin{aligned}
& {\left[\begin{array}{cc}
\Gamma & M_{i}^{T} \\
M_{i} & u_{\max }^{2} I
\end{array}\right] \geq 0,} \\
& \forall i, j=1, \ldots, r, i \leq j, \\
& {\left[\begin{array}{cc}
1 & x(0)^{T} \\
x(0) & \Gamma
\end{array}\right] \geq 0} \\
& \forall i=1, \ldots, r, \\
& \Gamma>0 \text {, }
\end{aligned}
$$


237

$$
\begin{aligned}
& \dot{x}^{T}(t) P x(t)+x^{T}(t) P \dot{x}(t) \\
& +\sum_{i=1}^{r} \sum_{j=1}^{r} h_{i}(s(t)) h_{j}(s(t)) x^{T}(t) C_{i}^{T} C_{j} x(t)-\gamma^{2} w^{T}(t) w(t) \\
& =\sum_{i=1}^{r} \sum_{j=1}^{r} h_{i}(s(t)) h_{j}(s(t)) x^{T}(t)\left(A_{i}-B_{i} F_{j}\right)^{T} P x(t) \\
& +\sum_{i=1}^{r} \sum_{j=1}^{r} h_{i}(s(t)) h_{j}(s(t)) x^{T}(t) P\left(A_{i}-B_{i} F_{j}\right) x(t) \\
& +\sum_{i=1}^{r} \sum_{j=1}^{r} h_{i}(s(t)) h_{j}(s(t)) x^{T}(t) C_{i}^{T} C_{j} x(t)-\gamma^{2} w^{T}(t) w(t) \\
& +\sum_{i=1}^{r} h_{i}(s(t)) x^{T}(t) E_{i}^{T} P x(t)+\sum_{i=1}^{r} h_{i}(s(t)) x^{T}(t) P E_{i} w(t) \\
& =\sum_{i=1}^{r} \sum_{j=1}^{r} h_{i}(s(t)) h_{j}(s(t))\left[\begin{array}{ll}
x^{T}(t) & w^{T}(t)
\end{array}\right] \\
& {\left[\begin{array}{cc}
\left(A_{i}-B_{i} F_{j}\right)^{T} P+P\left(A_{i}-B_{i} F_{j}\right)+C_{i}^{T} C_{j} & P E_{i} \\
E_{i}^{T} P & -\gamma^{2} I
\end{array}\right]\left[\begin{array}{c}
x(t) \\
w(t)
\end{array}\right] \leq 0 .}
\end{aligned}
$$




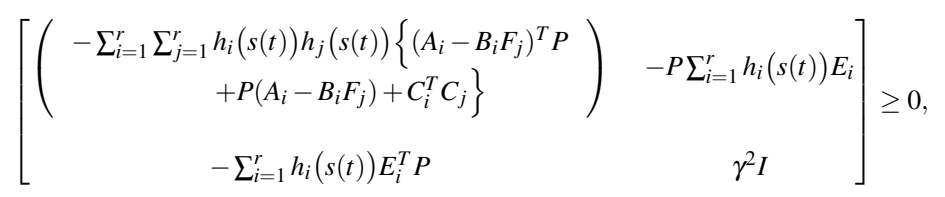

248

which can be decomposed as

$$
\left.\begin{array}{c}
{\left[\begin{array}{cc}
\left(\begin{array}{c}
\left.-\sum_{i=1}^{r} \sum_{j=1}^{r} h_{i}(s(t)) h_{j}(s(t))\right\} \\
\left.\left(A_{i}-B_{i} F_{j}\right)^{T} P+P\left(A_{i}-B_{i} F_{j}\right)\right\}
\end{array}\right] & -P \sum_{i=1}^{r} h_{i}(s(t)) E_{i} \\
-\sum_{i=1}^{r} h_{i}(s(t)) E_{i}^{T} P & \gamma^{2} I
\end{array}\right]} \\
-\left[\begin{array}{cc}
\sum_{i=1}^{r} \sum_{j=1}^{r} h_{i}(s(t)) h_{j}(s(t)) C_{i}^{T} C_{j} & 0 \\
0 & 0
\end{array}\right] \\
=\left[\begin{array}{cc}
-\sum_{i=1}^{r} \sum_{j=1}^{r} h_{i}(s(t)) h_{j}(s(t))\{ \\
\left.\left(A_{i}-B_{i} F_{j}\right)^{T} P+P\left(A_{i}-B_{i} F_{j}\right)\right\}
\end{array}\right) \\
-P \sum_{i=1}^{r} h_{i}(s(t)) E_{i} \\
-\sum_{i=1}^{r} h_{i}(s(t)) E_{i}^{T} P \\
-\left[\begin{array}{cc}
-\sum_{i=1}^{r} h_{i}(s(t)) C_{i}^{T} \\
0
\end{array}\right]\left[\begin{array}{cc}
{\left[\sum_{i=1}^{r} h_{i}(s(t)) C_{i}\right.} & 0
\end{array}\right] \geq 0 .
\end{array}\right]
$$

The inequality condition (34) is equivalent to

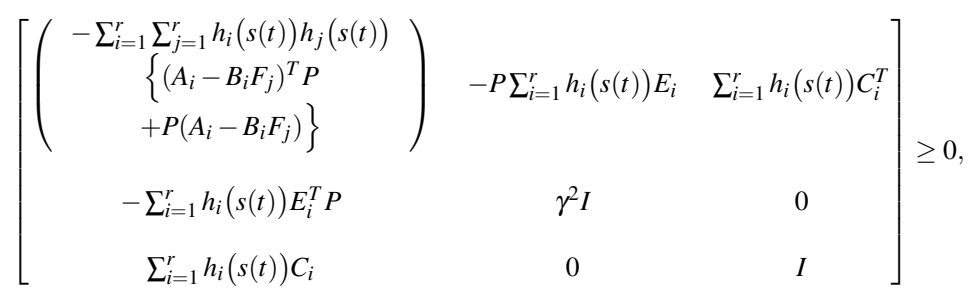

which can be rewritten as

$$
\begin{aligned}
& \sum_{i=1}^{r} \sum_{j=1}^{r} h_{i}(s(t)) h_{j}(s(t))
\end{aligned}
$$

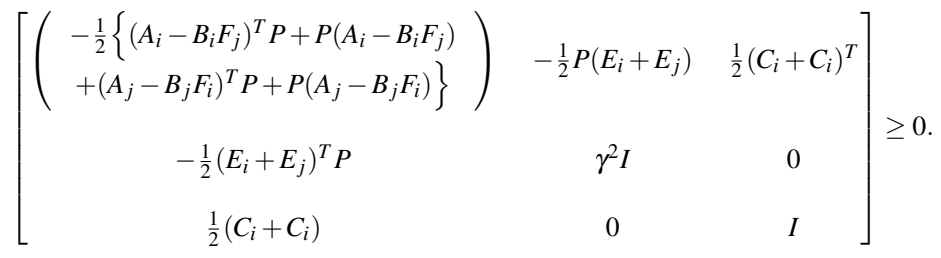




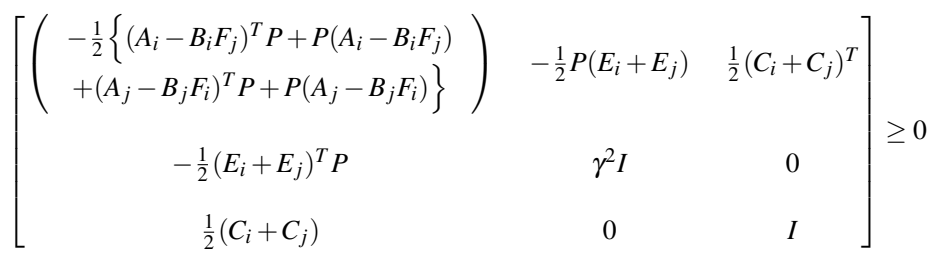

252

253

Now by multiplying the both side of equation (37) by block-diagonal $\{\Gamma I I\}$, LMI (27a) is obtained, where $\Gamma=P^{-1}$.

Part 2: To prove the bounding LMI condition (27b), from $\|u\|_{2} \leq u^{\max }$ one can write

$$
u^{T}(t) u(t)=\sum_{i=1}^{r} \sum_{j=1}^{r} h_{i}(s(t)) h_{j}(s(t)) x^{T}(t) F_{i}^{T} F_{j} x(t) \leq u_{\max }^{2},
$$

and then

$$
\frac{1}{u_{\max }^{2}} \sum_{i=1}^{r} \sum_{j=1}^{r} h_{i}(s(t)) h_{j}(s(t)) x^{T}(t) F_{i}^{T} F_{j} x(t) \leq 1 .
$$

Since $x^{T}(t) \Gamma^{-1} x(t)<x^{T}(0) \Gamma^{-1} x(0) \leq 1$ for all $t>0$, the inequality (39) holds if

$$
\frac{1}{u_{\max }^{2}} \sum_{i=1}^{r} \sum_{j=1}^{r} h_{i}(s(t)) h_{j}(s(t)) x^{T}(t) F_{i}^{T} F_{j} x(t) \leq x^{T}(t) \Gamma^{-1} x(t),
$$

and consequently (39) holds. Thus, we have

$$
\sum_{i=1}^{r} \sum_{j=1}^{r} h_{i}(s(t)) h_{j}(s(t)) x^{T}(t)\left(\frac{1}{u_{\max }^{2}} F_{i}^{T} F_{j}-\Gamma^{-1}\right) x(t) \leq 0 .
$$

The left-hand-side of (41) is equivalent to

$$
\begin{aligned}
\frac{1}{2} \sum_{i=1}^{r} \sum_{j=1}^{r} h_{i}(s(t)) h_{j}(s(t)) x^{T}(t)\left(\frac{1}{u_{\max }^{2}} F_{i}^{T} F_{j}+\frac{1}{u_{\max }^{2}} F_{j}^{T} F_{i}-2 \Gamma^{-1}\right) x(t) \\
=\frac{1}{2} \sum_{i=1}^{r} \sum_{j=1}^{r} h_{i}(s(t)) h_{j}(s(t)) x^{T}(t) \\
\quad\left(\frac{1}{u_{\max }^{2}}\left(F_{i}^{T} F_{j}+F_{j}^{T} F_{i}\right)-\frac{1}{u_{\max }^{2}}\left(F_{i}^{T}-F_{j}^{T}\right)\left(F_{i}-F_{j}\right)-2 \Gamma^{-1}\right) x(t) \\
\leq \frac{1}{2} \sum_{i=1}^{r} \sum_{j=1}^{r} h_{i}(s(t)) h_{j}(s(t)) x^{T}(t)\left(\frac{1}{u_{\max }^{2}}\left(F_{i}^{T} F_{j}+F_{j}^{T} F_{i}\right)-2 \Gamma^{-1}\right) x(t) \\
=\sum_{i=1}^{r} h_{i}(s(t)) x^{T}(t)\left(\frac{1}{u_{\max }^{2}} F_{i}^{T} F_{i}-\Gamma^{-1}\right) x(t) .
\end{aligned}
$$

Then, the inequality (41) holds if

$$
\frac{1}{u_{\max }^{2}} F_{i}^{T} F_{i}-\Gamma^{-1} \leq 0 \text {. }
$$

By defining $\mathbf{M}_{i}=\mathbf{F}_{i} \Gamma$, we have

$$
\frac{1}{u_{\max }^{2}} M_{i}^{T} M_{i}-\Gamma \leq 0 .
$$

and using the Schur Complement, the LMI condition (27b) is obtained. The proof is completed. The overall modeling steps and controller design stages are depicted in Figure 6. 


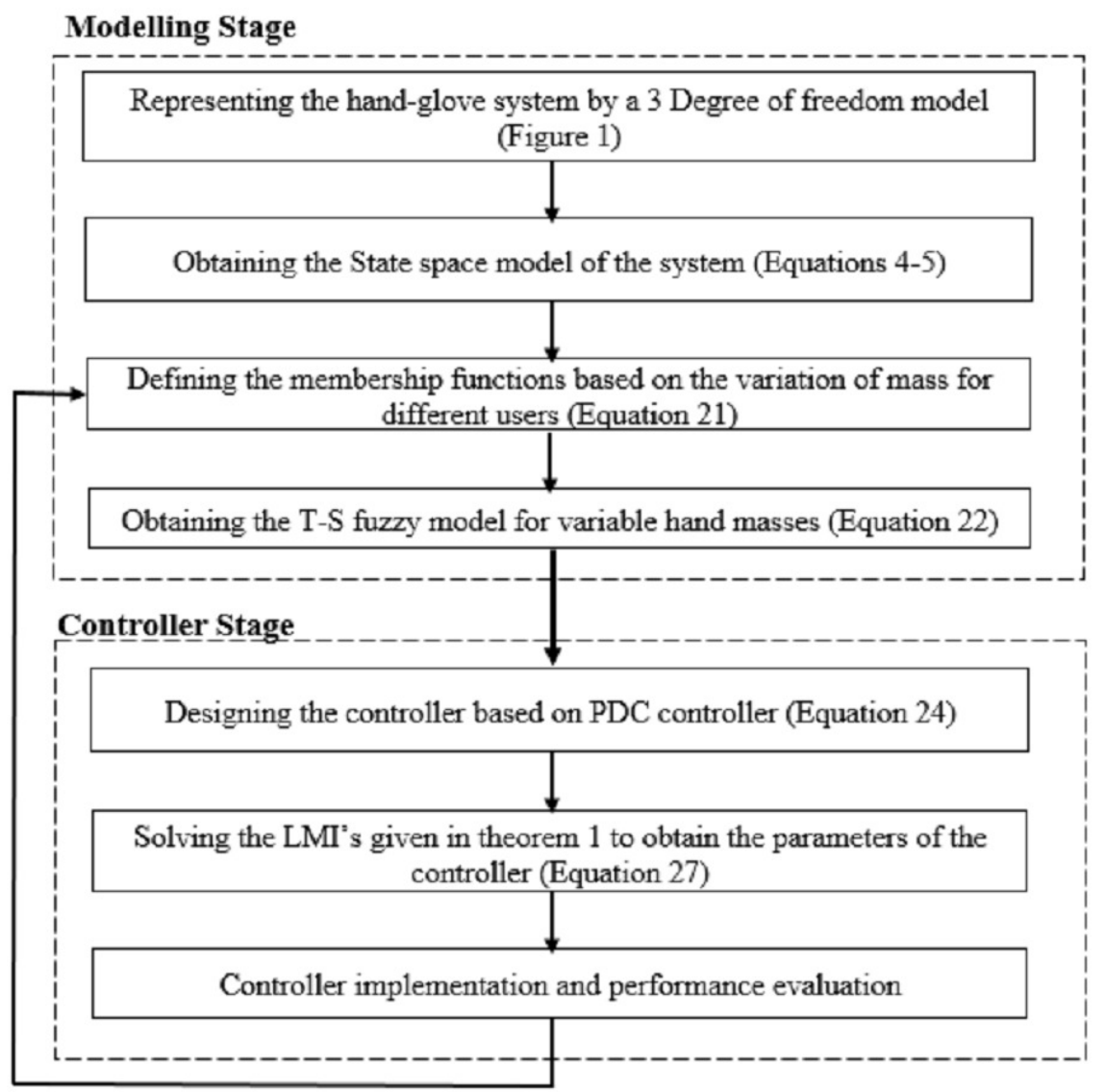

Figure 6. The overall design procedure

\section{SIMULATION RESULTS}

According to (Rezali and Griffin, 2018), the mass of the palm and fingers for any person can be estimated as a percentage of its whole body mass as represented in Table 2 . Thus, the total mass of the hand can be

\begin{tabular}{c|c} 
Segment & Mass $(\mathrm{kg})$ \\
\hline$m_{\text {palm }}$ & $0.75 \times 0.006 \times M_{\text {body }}$ \\
$m_{\text {fingers }}$ & $0.25 \times 0.006 \times M_{\text {body }}$
\end{tabular}

Table 2. Mass of the palm and fingers.

expressed as $m_{1}=0.006 \times M_{\text {body }}$. Here, by assuming that the body mass of a human that works with the hand-held devices may vary between $[50,90] \mathrm{kg}$, the minimum and maximum possible values of $m_{1}$ and $\psi_{h}$ based on Equation (18) are calculated and presented in Table 3. In the following, by considering the

\begin{tabular}{c|c|c} 
Parameter & Minimum & Maximum \\
\hline$m_{1}$ & 0.3 & 0.54 \\
$\psi_{h}$ & 1.85 & 3.33
\end{tabular}

Table 3. Minimum and maximum values of $m_{1}$ and $\psi_{h}$.

membership functions given in Equation (21) and by substituting the minimum and maximum values of $m_{1}$ from Table 3, they are obtained as in Equation (45) and represented in Figure 7.

$$
\begin{aligned}
& M_{1}\left(\psi_{h}(t)\right)=0.68 \psi_{h}(t)-1.25, \\
& M_{2}\left(\psi_{h}(t)\right)=-0.68 \psi_{h}(t)+2.25
\end{aligned}
$$




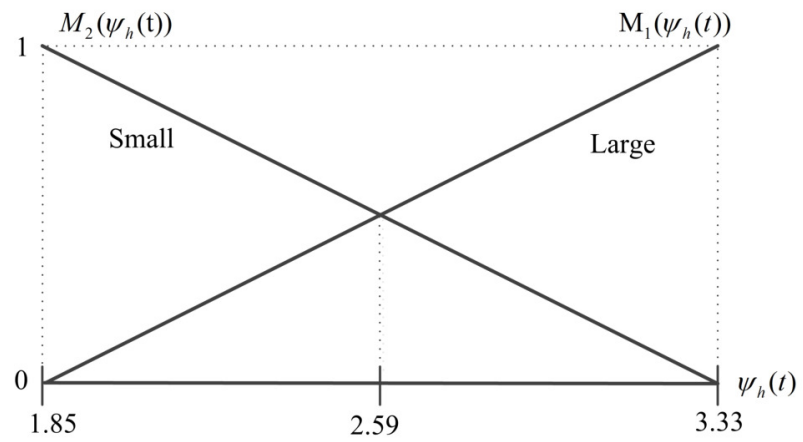

Figure 7. Membership Functions $M_{1}\left(\psi_{h}(t)\right)$ and $M_{2}\left(\psi_{h}(t)\right)$ for $\psi_{h}^{\min }=1.85$ and $\psi_{h}^{\max }=3.33$.

The stiffness and damping coefficients of the hand-glove system of Figure 1 are depicted in Table 4.

\begin{tabular}{c|c|c|c} 
Parameter & Value & Parameter & Value \\
\hline$m_{1}^{\min }$ & $0.3 \mathrm{Kg}$ & $c_{2}$ & $36 \mathrm{~N} / \mathrm{m}$ \\
$m_{1}^{\max }$ & $0.54 \mathrm{Kg}$ & $c_{3}$ & $1.53 \mathrm{~N} / \mathrm{m}$ \\
$m_{2}$ & $0.01 \mathrm{Kg}$ & $k_{1}$ & $0.01 \mathrm{~N} . \mathrm{s} / \mathrm{m}$ \\
$m_{3}$ & $0.078 K g$ & $k_{2}$ & $3667 N . s / m$ \\
$c_{1}$ & $1 \mathrm{~N} / \mathrm{m}$ & $k_{3}$ & $1152 N . s / m$
\end{tabular}

Table 4. The parameter's values used in simulations.

\subsection{The Hand-Glove System Representation Based on the Fuzzy T-S Model with PDC} controller

By considering the T-S fuzzy model (22) and the fuzzy rules (24) derived for the hand-glove system with variable hand masses and by assuming the maximum and minimum hand masses of $m_{1}^{\min }=0.3 \mathrm{Kg}$, $m_{1}^{\max }=0.54 \mathrm{Kg}$, respectively, the associated subsystems based on the Equation (23) are derived as follows:

$$
\begin{aligned}
& A_{1}=\left[\begin{array}{cccccc}
0 & 0 & 0 & 1 & 0 & 0 \\
0 & 0 & 0 & 0 & 1 & 0 \\
0 & 0 & 0 & 0 & 0 & 1 \\
-12223.37 & 12223.33 & 0 & -123.33 & 120 & 0 \\
366700 & -481900 & 115200 & 3600 & -3753 & 153 \\
0 & 2035.33 & -2035.33 & 0 & 2.70 & -2.70
\end{array}\right] \\
& A_{2}=\left[\begin{array}{cccccc}
0 & 0 & 0 & 1 & 0 & 0 \\
0 & 0 & 0 & 0 & 1 & 0 \\
0 & 0 & 0 & 0 & 0 & 1 \\
-6790.76 & 6790.74 & 0 & -68.52 & 66.67 & 0 \\
366700 & -481900 & 115200 & 3600 & -3753 & 153 \\
0 & 2035.33 & -2035.33 & 0 & 2.70 & -2.70
\end{array}\right] \\
& B_{1}=B_{2}=\left[\begin{array}{cccccc}
0 & 0 & 0 & 0 & 100 & -1.77 \\
0 & 0 & 0 & 0 & -100 & 0
\end{array}\right]^{T} \\
& E_{1}=E_{2}=\left[\begin{array}{llllll}
0 & 0 & 0 & 0 & 0 & 1.77
\end{array}\right]^{T} \\
& C_{1}=C_{2}=\left[\begin{array}{llllll}
0 & 0 & 0 & 1 & 0 & 0
\end{array}\right] .
\end{aligned}
$$


In the simulation work, the vibration source is assumed to be a 2-stroke engine that is based on (Ko et al., 2011), producing a vibration given by the sum of a white noise signal and sine waves with frequencies of 50,80 and $100 \mathrm{~Hz}$ and amplitude of 8,5 and 3, respectively. The input disturbance to the system and its frequency spectrum is shown in Figure 8 (A) and (B), respectively. As can be seen in Figure 8 (B) the engine has the highest peak at $50 \mathrm{~Hz}$ with a magnitude of $5.5 \mathrm{~m} / \mathrm{s}^{2}$.

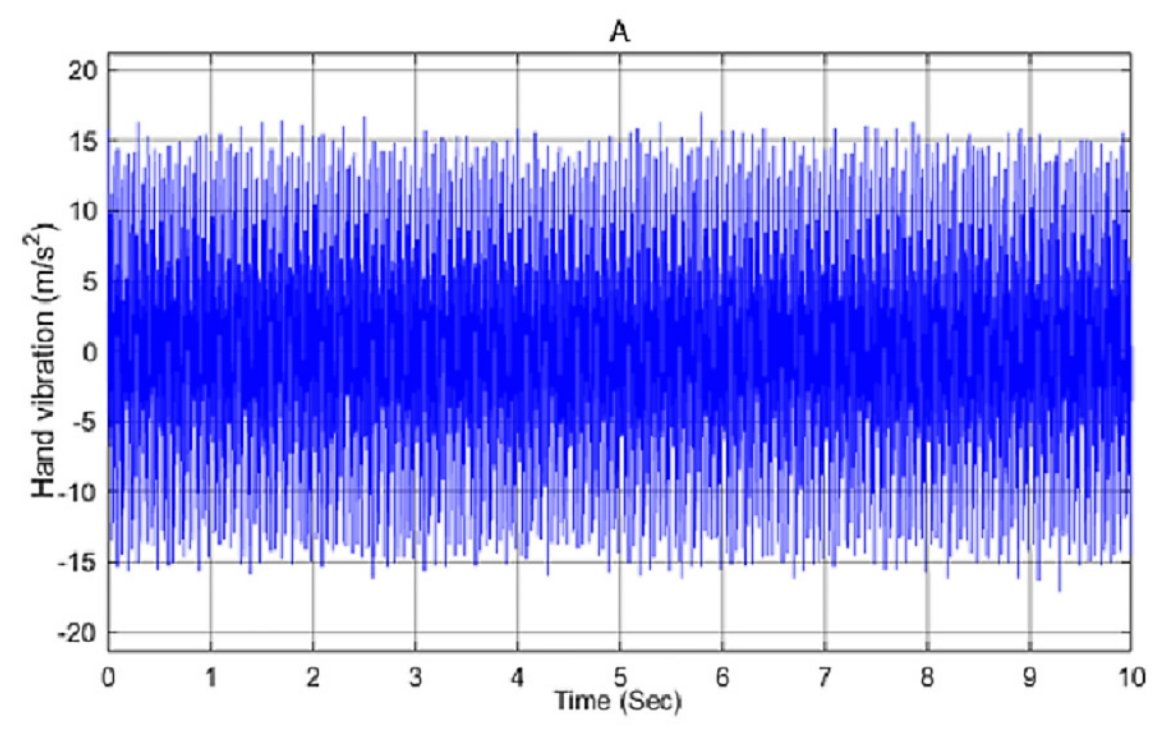

B

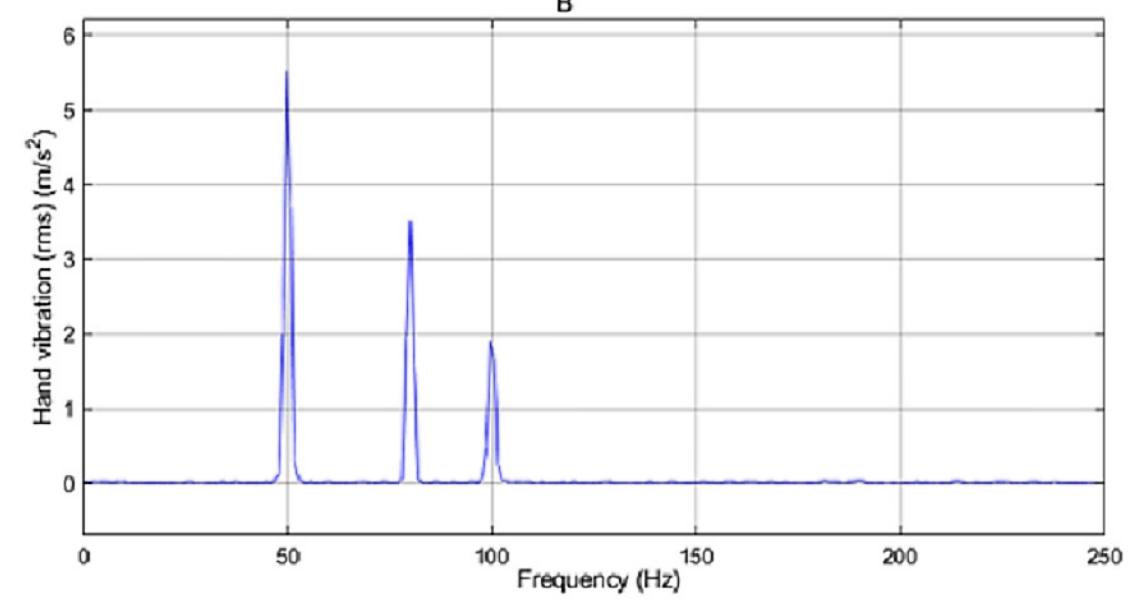

Figure 8. (A) Input disturbance to the system and (B) Frequency spectra of the input vibration

The amount of vibration received by the user in the passive mode i.e. no controller is applied to the system is represented in Figure 9. By applying the input disturbance to the system without using any controller, the hand vibration decreased to under $10 \mathrm{~m} / \mathrm{s}^{2}$ but still, it is not in the healthy range for humans. As shown in Figure 9 (B), the vibration spectra peak related to the hand acceleration in the passive model is reduced to $2.5 \mathrm{~m} / \mathrm{s}^{2}$.

\subsection{Applying the Proposed Fuzzy Robust PDC Controller}

In the hand-glove fuzzy system (46), the mass of the hand is considered to be $0.4 \mathrm{~kg}$ and values of $m_{1}^{\text {min }}$ and $m_{1}^{\max }$ is chosen to be 0.3 and $0.54 \mathrm{~kg}$, respectively (Table 3). By considering the system matrices in (46) and solving the LMIs in Theorem 1, which were implemented using MATLAB R2018a and YALMIP (Lofberg, 2004) and solved using MOSEK 8, the values of the feedback gains of the fuzzy PDC controller $\left(F_{1}\right.$ and $\left.F_{2}\right)$ and the $P$ matrix are obtained as follows: 

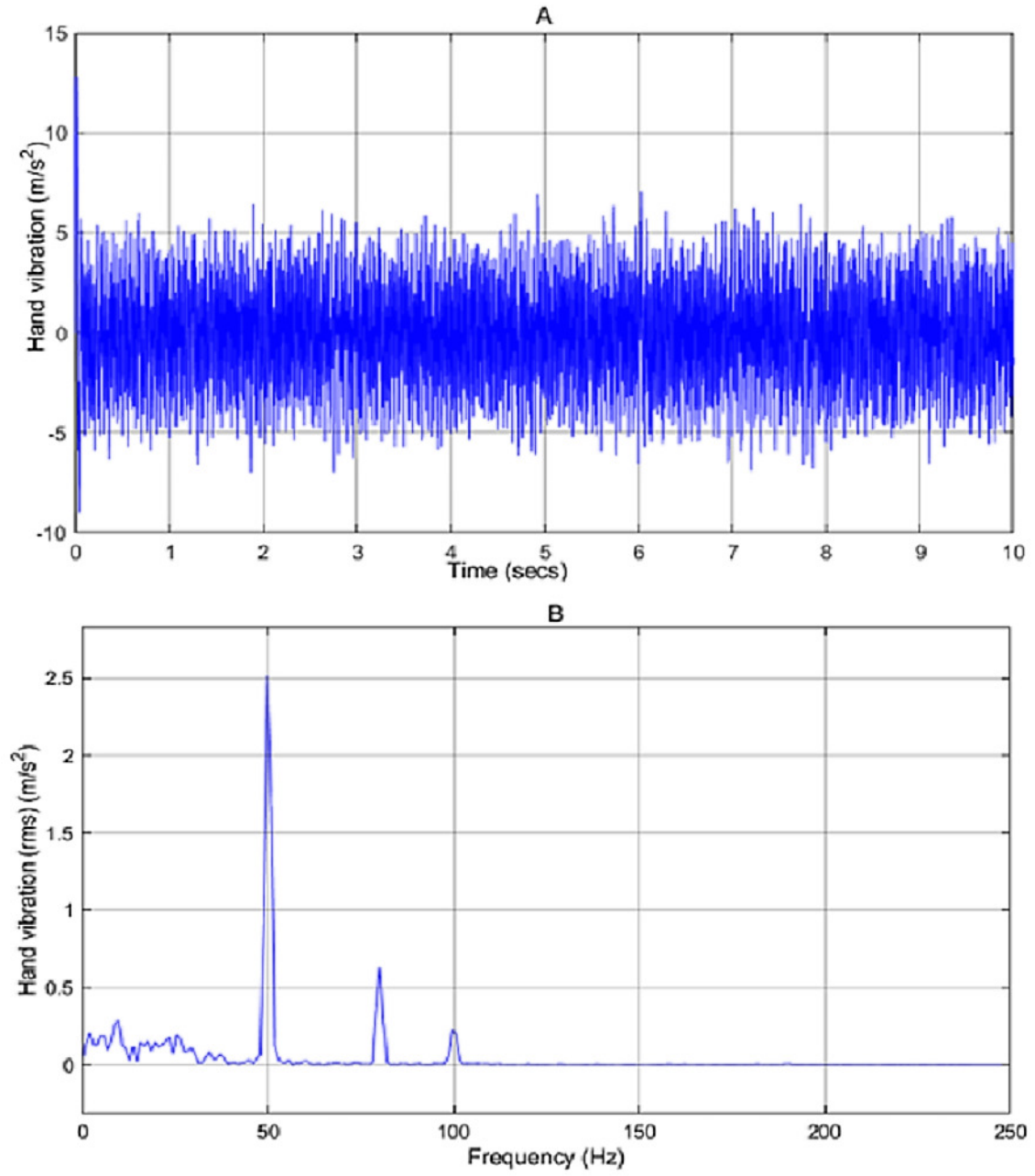

Figure 9. Vibration of the passive model. (A) The hand vibration in passive model, without applying a controller, (B) Frequency spectra of the hand vibration in passive model

$$
\begin{aligned}
F_{1} & =\left[\begin{array}{lllllll}
-7.847 & 9.661 & 4.779 & 25.148 & 572.073 & -0.299
\end{array}\right] \\
F_{2} & =\left[\begin{array}{lllllll}
-5.412 & 12.267 & 0.208 & 16.006 & 321.739 & -0.407
\end{array}\right] \\
P & =\left[\begin{array}{ccccccc}
14.685 & -7.641 & -2.492 & 1.784 & -0.035 & 0.224 \\
-7.643 & 7.925 & -0.183 & -0.047 & 0.067 & 0.026 \\
-2.492 & -0.183 & 2.052 & -0.393 & 0.006 & 0.045 \\
1.784 & -0.047 & -0.393 & 0.586 & 0.010 & 0.081 \\
-0.035 & 0.067 & 0.006 & 0.010 & 0.001 & 0.005 \\
0.224 & 0.026 & 0.045 & 0.081 & 0.005 & 0.040
\end{array}\right]
\end{aligned}
$$

The above feedback gains $F 1$ and $F 2$ give positive eigenvalues of $P$ that satisfy the positive definiteness of $P$ required in the LMI (27d) of Theorem 1.

By applying the designed fuzzy PDC controller to the hand-glove system (46), the results are as shown in Figure 10. By comparing the obtained results with the PID and AFC controllers, it can be seen that the designed fuzzy PDC controller has a much better performance. As shown in Figure 10 (A), we can see that the proposed fuzzy PDC controller has reduced the vibration experienced by the user to around $0.4 \mathrm{~m} / \mathrm{s}^{2}$, well within the healthy zone $\left(<2.5 \mathrm{~m} / \mathrm{s}^{2}\right)$, defined by the international standards. 

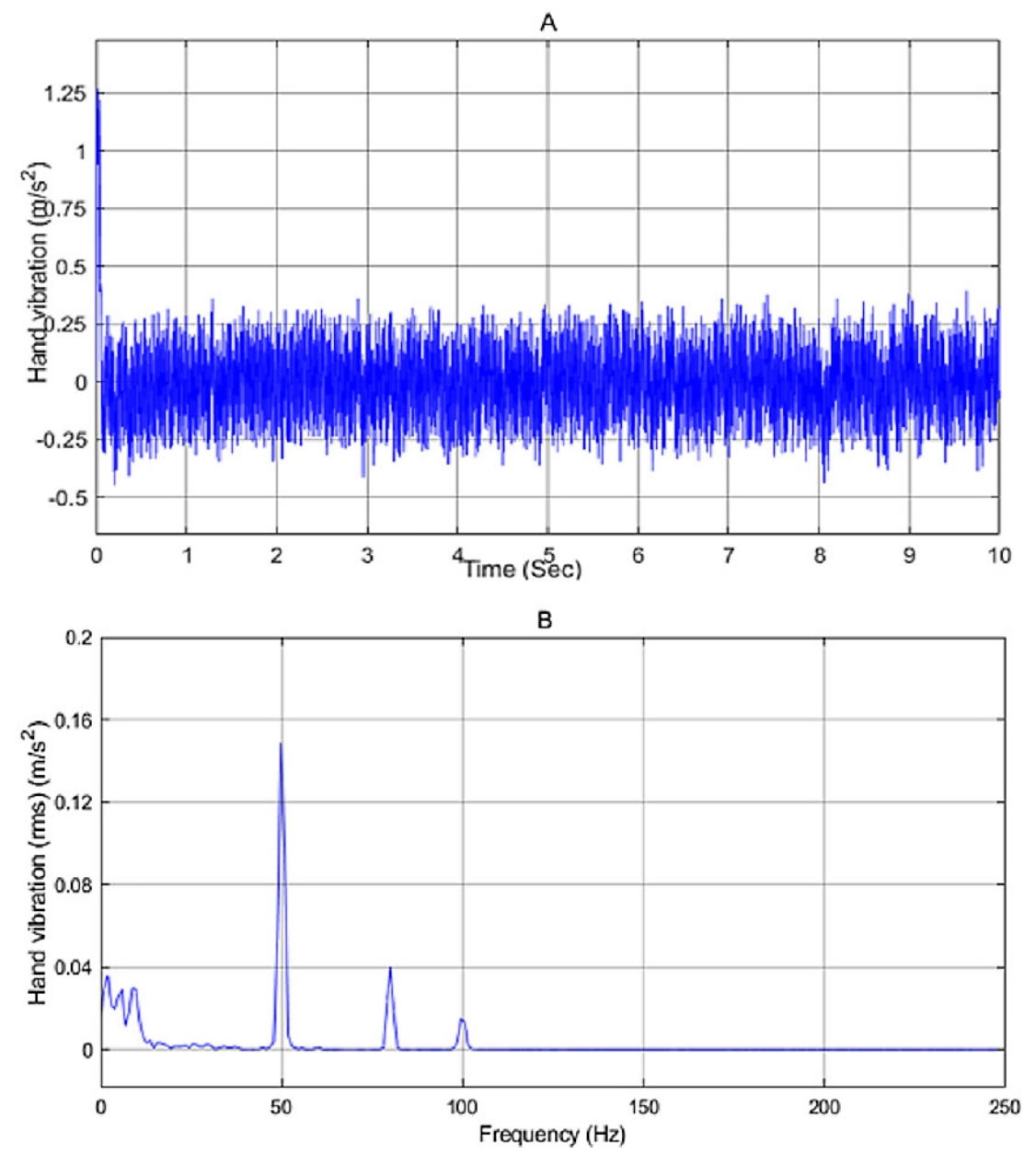

Figure 10. System vibration with PDC controller. (A) The vibration at the hand after applying PDC controller (B) The frequency spectra of the hand vibration after applying PDC controller

One can clearly see the excellent performance of the proposed fuzzy PDC controller in suppressing the vibration compared to the other active vibration controllers as illustrated in Figure 11. From the simulation results, it can be seen that the proposed controller has a good vibration rejection mechanism.

To show the robustness of the proposed controller to the variation of the hand masses $m_{1}$, we have changed the hand mass of hand $m_{1}$ from $0.4 \mathrm{~kg}$ to $0.5 \mathrm{~kg}$. As shown in Figure 12, the amplitude of the hand vibration has only a small variation when compared with Figure 10 (A) and is still within the healthy range for humans.

\section{CONCLUSION}

In this paper, a biodynamic model of the hand-glove system was developed based on the available models for the hand-glove system but focusing more on the vibration experienced by the hand. Then, a robust fuzzy PDC controller was proposed to minimize the vibration transmitted to the hand. By applying the designed controller to the hand-glove system, the amount of vibration experienced by the hand reduced to around $0.4 \mathrm{~m} / \mathrm{s}^{2}$ well within a healthy vibration range. Figure 13 summarizes the performances of the different controllers for the hand-glove system experiencing vibrations from vibrating tool usage. The vibrations transferred to the hand using the proposed fuzzy PDC were $93 \%$ and $85 \%$ less compared to the PID controller and the active force controller (AFC), respectively. Also, the proposed controller was robust to the changes of hand masses. 


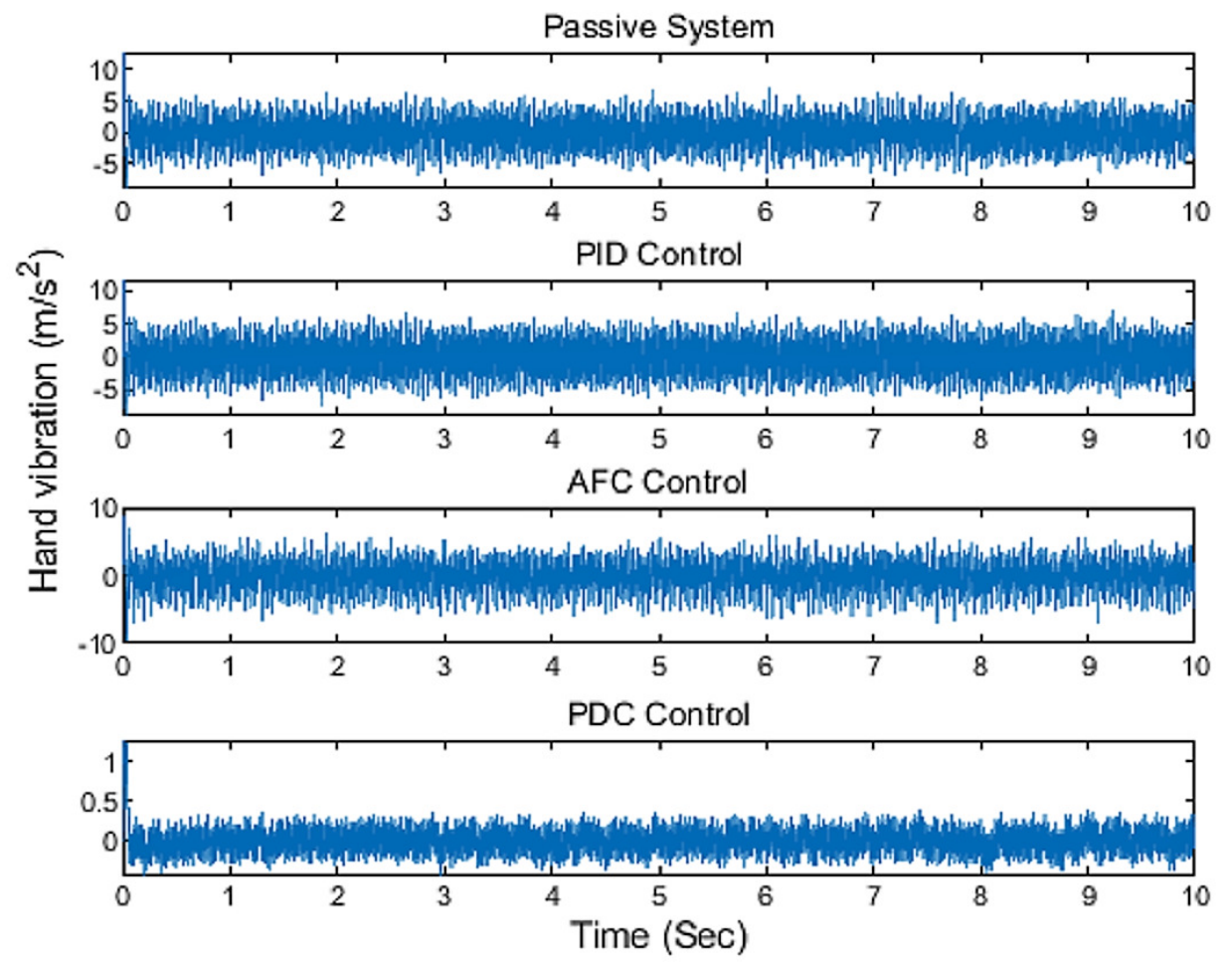

Figure 11. The comparison result of applying different controllers

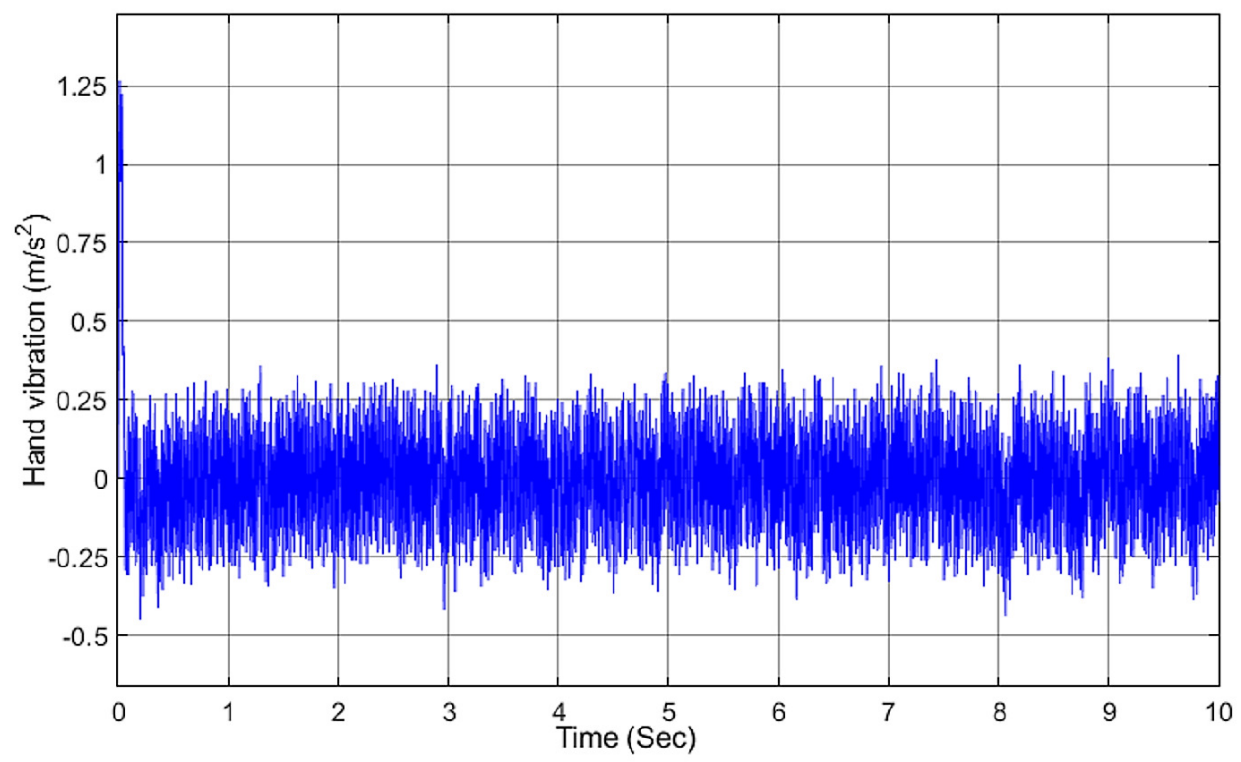

Figure 12. Vibration of the hand with PDC controller while changing $m_{1}$

\section{REFERENCES}

Abdelmaksoud, S. I., Mailah, M., and Abdallah, A. M. (2020). Robust intelligent self-tuning active force control of a quadrotor with improved body jerk performance. IEEE Access, 8:150037-150050.

Chen, L., Li, X., Xiao, W., Li, P., and Zhou, Q. (2019). Fault-tolerant control for uncertain vehicle active 


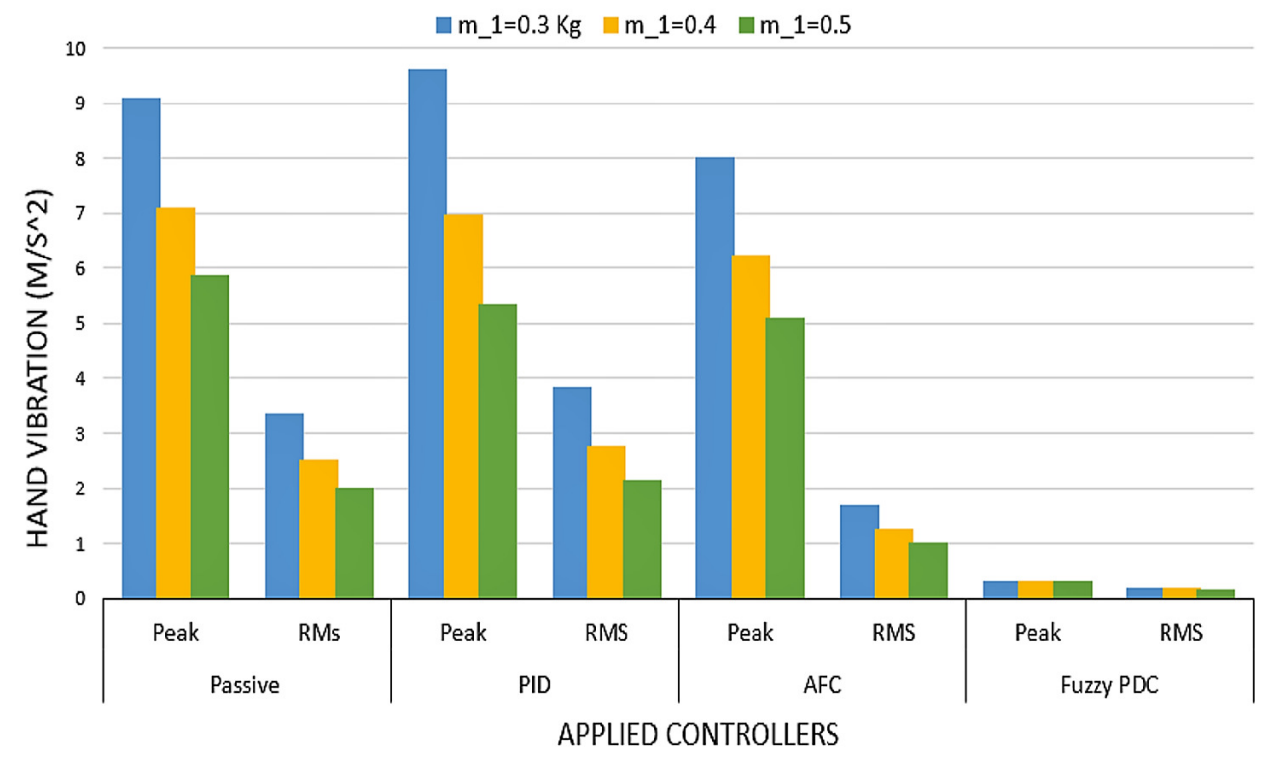

Figure 13. Hand vibration of varying hand masses and different controllers

steering systems with time-delay and actuator fault. International Journal of Control, Automation and Systems, 17(9):2234-2241.

Dong, R. G., McDowell, T. W., Welcome, D. E., Warren, C., Wu, J. Z., and Rakheja, S. (2009). Analysis of anti-vibration gloves mechanism and evaluation methods. Journal of Sound and Vibration, 321(12):435-453.

Dong, R. G., Sinsel, E. W., Welcome, D. E., Warren, C., Xu, X. S., McDowell, T. W., and Wu, J. Z. (2015). Review and evaluation of hand-arm coordinate systems for measuring vibration exposure, biodynamic responses, and hand forces. Safety and health at work, 6(3):159-173.

Gao, S. and Liu, J. (2020). Adaptive fault-tolerant vibration control of a wind turbine blade with actuator stuck. International Journal of Control, 93(3):713-724.

Gerhardsson, L., Ahlstrand, C., Ersson, P., and Gustafsson, E. (2020). Vibration-induced injuries in workers exposed to transient and high frequency vibrations. Journal of Occupational Medicine and Toxicology, 15(1):1-9.

Gohari, M. and Tahmasebi, M. (2017). Comparative study between intelligent algorithms for active force control of side car mirror vibration. International Journal of Control Science and Engineering 2017, pages 32-43.

Hamouda, K., Rakheja, S., Marcotte, P., and Dewangan, K. (2017). Fingers vibration transmission performance of vibration reducing gloves. International Journal of Industrial Ergonomics, 62:55-69.

Hamzah, N., Aripin, M. K., Sam, Y. M., Selamat, H., and Ghazali, R. (2012). Second order sliding mode controller for longitudinal wheel slip control. In 2012 IEEE 8th International Colloquium on Signal Processing and its Applications, pages 138-143.

Hassan, M., Mailah, M., Junid, R., and Alang, N. (2010). Vibration suppression of a handheld tool using intelligent active force control (afc). In Proceedings of the World Congress on Engineering, volume 2, pages 1636-1641.

Jones, D. I. (2001). Handbook of viscoelastic vibration damping. John Wiley \& Sons.

Kalaivani, R., Sudhagar, K., and Lakshmi, P. (2016). Neural network based vibration control for vehicle active suspension system. Indian Journal of Science and Technology, 9(1).

Kamalakar, G. B. and Mitra, A. C. (2018). Development and analysis of human hand-arm system model for anti-vibration isolators. Materials Today: Proceedings, 5(2, Part 1):3943-3952. 7th International Conference of Materials Processing and Characterization, March 17-19, 2017.

Ko, Y. H., Ean, O. L., and Ripin, Z. M. (2011). The design and development of suspended handles for reducing hand-arm vibration in petrol driven grass trimmer. International Journal of Industrial Ergonomics, 41(5):459-470. 
Lekshmi, A. and Ramachandran, K. (2019). Parkinson's tremor suppression using active vibration control method. In IOP Conference Series: Materials Science and Engineering, volume 577, page 012056. IOP Publishing.

Li, Y., Wang, X., Wang, L., Fan, W., and Qiu, Z. (2017). Non-probabilistic stability reliability measure for active vibration control system with interval parameters. Journal of Sound and Vibration, 387:1-15.

Lin, B., Su, X., and Li, X. (2019). Fuzzy sliding mode control for active suspension system with proportional differential sliding mode observer. Asian Journal of Control, 21(1):264-276.

Liu, Y.-J., Zeng, Q., Liu, L., and Tong, S. (2018). An adaptive neural network controller for active suspension systems with hydraulic actuator. IEEE Transactions on Systems, Man, and Cybernetics: Systems.

Lofberg, J. (2004). Yalmip: A toolbox for modeling and optimization in matlab. In 2004 IEEE International Symposium on Computer Aided Control Systems Design, pages 284-289. IEEE.

Mailah, M., Jahanabadi, H., Zain, M., and Priyandoko, G. (2009). Modelling and control of a human-like arm incorporating muscle models. Proceedings of the Institution of Mechanical Engineers, Part C: Journal of Mechanical Engineering Science, 223(7):1569-1577.

Mazlan, A. Z. A. and Ripin, Z. M. (2015). Active vibration control to attenuate hand-arm vibration from orbital sander: A mathematical model approach. Indian Journal of Science and Technology, 8(30).

Miljković, D. (2009). Review of active vibration control. 32nd Int. Conv. on Info. and Comm. Tech., Electronics and Microelectronics, pages 103-108.

Mohamad, M., Mailah, M., and Muhaimin, A. (2006). Vibration control of mechanical suspension using active force control. In Procs. of Regional Conference on Vehicle Engineering Technology, RiVET.

Nguyen, A.-T., Dambrine, M., and Lauber, J. (2016). Simultaneous design of parallel distributed output feedback and anti-windup compensators for constrained takagi-sugeno fuzzy systems. Asian Journal of Control, 18(5):1641-1654.

Preumont, A. (2018). Vibration control of active structures: an introduction, volume 246. Springer.

Rajabpour, L., Shasadeghi, M., and Barzegar, A. (2019). Design of robust h8 fuzzy output feedback controller for affine nonlinear systems: fuzzy lyapunov function approach. International Journal of Advanced Intelligence Paradigms, 14(3-4):328-344.

Rani, M. R., Selamat, H., Zamzuri, H., and Ahmad, F. (2011). Pid controller optimization for a rotational inverted pendulum using genetic algorithm. In 2011 Fourth International Conference on Modeling, Simulation and Applied Optimization, pages 1-6.

Rezali, K. A. M. and Griffin, M. J. (2018). Transmission of vibration through glove materials: effects of contact force. Ergonomics, 61(9):1246-1258.

Sadeghi, M. S., Rezaei, M., and Mardaneh, M. (2015). Affine parallel distributed compensator design for affine fuzzy systems via fuzzy lyapunov function. Engineering Applications of Artificial Intelligence, 37:407-416.

Sadeghi, M. S., Safarinejadian, B., and Farughian, A. (2014). Parallel distributed compensator design of tank level control based on fuzzy takagi-sugeno model. Applied Soft Computing, 21:280-285.

Sadeghi, M. S., Vafamand, N., and Khooban, M. H. (2016). Lmi-based stability analysis and robust controller design for a class of nonlinear chaotic power systems. Journal of the Franklin Institute, 353(13):2835-2858.

Shen, S. C. and House, R. A. (2017). Hand-arm vibration syndrome: What family physicians should know. Canadian Family Physician, 63(3):206-210.

Sun, H., Li, Y., Xu, K., and Tong, S. (2018). Fuzzy adaptive backstepping control for a class of active suspension systems. IFAC-PapersOnLine, 51(31):136-141.

Tabatabaei, S., Barzegar, A., Sadeghi, M. S., and Roosta, P. (2010). Fuzzy pd+ i and fuzzy pid controllers design for a nonlinear quarter car suspension system. In 2010 International Conference on Computer Applications and Industrial Electronics, pages 186-190. IEEE.

Tahoun, A. (2017). A new online delay estimation-based robust adaptive stabilizer for multi-input neutral systems with unknown actuator nonlinearities. ISA transactions, 70:139-148.

Tahoun, A. H. (2020). Fault-tolerant control for a class of quantised networked control of nonlinear systems with unknown time-varying sensor faults. International Journal of Control, 93(3):619-628.

Tanaka, K. and Wang, H. O. (2004). Fuzzy control systems design and analysis: a linear matrix inequality approach. John Wiley \& Sons.

Theik, C. C. and Mazlan, A. Z. A. (2020). Active vibration control of an inertia-type piezoelectric actuator 
based suspended handle using pid-afc controller. In 2020 IEEE Symposium on Industrial Electronics \& Applications (ISIEA), pages 1-5. IEEE.

Vihlborg, P., Bryngelsson, L., Lindgren, B., Gunnarsson, L. G., and Graff, P. (2017). Association between vibration exposure and hand-arm vibration symptoms in a swedish mechanical industry. International Journal of Industrial Ergonomics, 62:77-81.

Wang, H. O., Tanaka, K., and Griffin, M. (1995). Parallel distributed compensation of nonlinear systems by takagi-sugeno fuzzy model. In Proceedings of 1995 IEEE International Conference on Fuzzy Systems., volume 2, pages 531-538. IEEE.

Zain, M. Z. M., Mailah, M., and Priyandoko, G. P. G. (2008). Active force control with input shaping technique for a suspension system. Jurnal Mekanikal, 27(2).

Zuo, G. and Wong, L. (2016). A review on recent active vibration control techniques. arXiv preprint arXiv:1601.05889. 